\title{
MAC Performance of a 3GPP-LTE Multihop Cellular Network
}

\author{
Rainer Schoenen, Ruediger Halfmann, and Bernhard H. Walke
}

\begin{abstract}
Multihop cellular networks offer increased coverage and capacity compared to singlehop cells of the same size. This is not as huge as using more base stations (BS) instead of wireless relay nodes (RN), but by this way there is no need for a fixed network access to each node. Therefore a cost-efficient deployment is easily achieved (less CAPEX and OPEX). Even when the coverage of a BS is already quite good, relays help to increase the overall system capacity.

This paper analyzes numerically with analytic background the multihop operation in the frequency division duplex mode and discusses the MAC-layer protocol performance for the 3GPPLTE [1] system. OFDMA with centrally controlled resource coordination is assumed here. By following the analytic model we show that for relaying, OFDMA resources are utilized effectively in time and frequency and provide good coverage and capacity results. Parameters like directional relay antennas, space or time division multiple access and relay antenna gain are studied.
\end{abstract}

Index Terms-Multihop, Relaying, FDD, LTE, SDM

\section{INTRODUCTION}

$\mathbf{M}$ ULTIHOP or Relaying is rather new for LTE [1]. In contrast to adhoc networks, fixed relays are placed at positions that were planned in advance. They provide improved coverage and capacity of the relay enhanced radio cells. That is why they are proposed for standards of next generation cellular systems. The demand for high data rates in large areas is omnipresent, but the offer of conventional cellular architectures does not match this demand. First, the limited power problem: For a given transmit power level, the higher transmission rates lead to a lower energy per bit. Second, the radio propagation: Above a few $\mathrm{GHz}$ the vulnerability to bad non-line-of-sight conditions is higher. In effect, the path loss is higher between base station and mobile. Third, the maximum data rate offered by a base station depends on the distance of the mobile to the base station, but the demand density is constant. Close to the base station a higher SINR value is achieved, which allows the highest Modulation\&Coding scheme (PhyMode) and therefore the highest data rate. At the cell edge the offered data rate is one order of magnitude lower $\left(Q P S K \frac{1}{3}\right.$ compared to $Q A M 64 \frac{5}{6}$ for LTE [1]). A transmission of a certain data rate therefore requires ten times more radio resources at the edge than near the BS. For this reason, the average cell capacity is heavily determined by the PhyModes at the cell border.

- Ruediger Halfmann is with NSN, Munich, Germany

- The other authors are with the Chair of Communication Networks at RWTH Aachen University, Faculty 6, Germany

- This work has been funded by the BMBF in Germany in the ScaleNet project
While placing more base stations (BS) per area seems to be a solution, this comes with much higher costs due to the fixed network access by fiber. In this paper we consider the deployment of Fixed Relay Stations, also called Relay Nodes (RN), which are fed over the same wireless technology. The cellular basic structure stays ideally hexagonal, if we place RNs within the original cell (Figure 1) in order to improve the capacity of the cell. The basic structure changes to three hexagons if we place RNs at the former border, so now there are hexagons around the three RNs and one BS at the intersection of the three hexagons. Sectorization of antennas is an additional way to reduce interference to neighbor cells. Cluster orders (frequency reuse factors) of three to twelve are common to have a guard distance between interfering cells. We can even utilize a spatial reuse among the RNs, i.e. let RNs transmit in parallel into different segments. Directional antennas at the RNs can then avoid any interference into the peer segments. In this paper we do not consider MIMO techniques, but their use is independent of relaying.

With OFDM the use of radio resources can be handled efficiently. OFDMA scheduling for multiple access is performed in the BS and decides the parameters to use for each resource unit [2], e.g. the best subcarrier for a user terminal, the PhyMode and the RF power per subcarrier, all adaptively. In the literature relaying appeared first for TDD systems [3], back to very early systems [4]. FDD and LTE [1] Multihop has been treated in [5], and there is also an extension for hald-duplex FDD [6]. In this paper we focus on performance results, and study the impact of parameters on the system.

The paper is organized to first define the relaying principles and the FDD specific terms. Next, the opportunities provided by OFDMA and cellular parameters are explained. The last section presents performance results for the various parameters. The paper ends with a concluding summary.

\section{THEORY OF RELAYING}

Relay Nodes in this context are used as repeaters, bridges or routers, i.e. they operate at layer-2, in contrast to simple amplify-and-forward nodes on layer- 1 . Here, received PDUs are fully error-corrected, automatically repeated (ARQ), stored and scheduled in the RN for further transmission, and if necessary even segmented and reassembled prior to their next-hop transmission. The medium access control (MAC) is in charge of introducing the relay resources into the frame structure of an existing protocol. For relaying we basically have the (transparent) subframe concept [7] and the (relayaware) resource reservation concept which we follow here. 


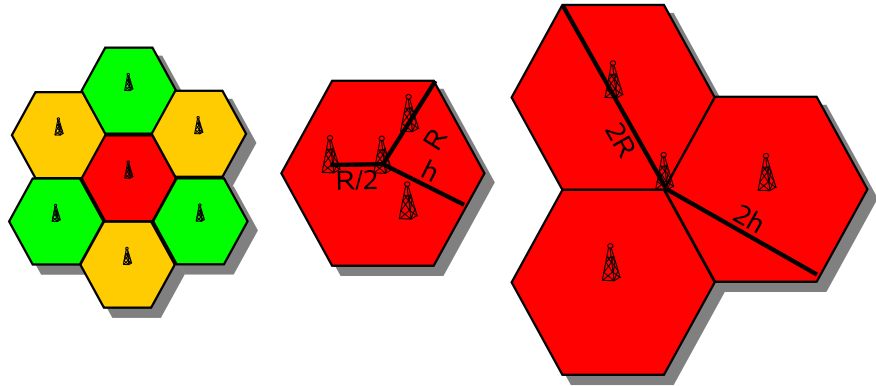

Fig. 1. Left: Conventional cellular geometry, Middle: Geometry to increase capacity, Right: Geometry to increase coverage

For the UT the RN acts like a BS on the second (last) hop (transparent). On the first hop, where the RN acts like a UT towards the BS, extra resources are required.

\section{A. Downlink and Uplink Frame Format}

We assume two hops here, but there is no principal limitation. Traffic PDUs are transmitted by the BS to the RN using dedicated DL resource blocks. For the BS, the RN is acts like a UT. On the second hop, there are resources required between $\mathrm{RN}$ and UT. These resources are coarsely assigned by the BS, e.g. a certain number of reserved subchannels. These resources can be subdivided by the RN with full freedom of choice. Like a BS, the RN decides which resource to use for which UT.

Due to the different $S I N R$ situation on the first and second hop, a different PhyMode is chosen based on the values in Table I. In OFDMA, this PhyMode can be different for each subchannel, depending on the frequency-dependent condition of the fading channel. Therefore, for one Multihop transmission, the size of the resources needed for the second hop differs from that of the first hop. Thus it is important that the RN decides on the PhyModes itself and schedules the PDUs to be forwarded on its own. On the last hop, UTs receive PDUs from $\mathrm{RN}$ just like they would from a $\mathrm{BS}$, i.e. the do not need to be relay aware.

The uplink (UL) works such that an UT announces any available traffic to its superior station, which is the RN. The RN assigns UL resources to UT for the PDU transmission while it also announces this new uplink traffic towards the BS. PDUs received in the uplink of hop 2 are stored in RN until the UL resources are available for hop 1 . So the BS does not need to know what is going on exactly on hop2. It only needs to know that there is a big resource block reserved for the use in hop 2 .

Figure 1 shows two characteristic scenarios for the use of relays. Let the cluster order be $C$. In the coverage extension scenario, the supported hexagonal area around relay nodes is of same size as the singlehop area around the base station. The inner hexagon is typically served by BS within its range, while the outer regions $\left(+3 \cdot \frac{2}{3}=200 \%\right)$ are served by one of the three RNs respectively. The frequency reuse distance is

$$
D=3 R \cdot \sqrt{C} \text {. }
$$

For capacity extension, the whole hexagonal area can be served

\begin{tabular}{|l|l|l|l|}
\hline PhyMode & modulation & code rate & $S I N R_{\min }$ in $[d B]$ \\
\hline 1 & QPSK & $1 / 3$ & 0.9 \\
2 & QPSK & $1 / 2$ & 2.1 \\
3 & QPSK & $2 / 3$ & 3.8 \\
4 & QAM16 & $1 / 2$ & 7.7 \\
5 & QAM16 & $2 / 3$ & 9.8 \\
6 & QAM16 & $5 / 6$ & 12.6 \\
7 & QAM64 & $2 / 3$ & 15.0 \\
8 & QAM64 & $5 / 6$ & 18.2 \\
\hline
\end{tabular}

TABLE I

LTE PhyModes AND THEIR REQUIRED $S I N R_{\text {min }}$

by the BS. Here the frequency reuse distance is

$$
D=R \cdot \sqrt{3 C} \text {. }
$$

In regions closer to the cell border and near the $\mathrm{RN}$, the $\mathrm{RNs}$ offer a much higher data rate to UTs than the BS would. Even more strictly, there are areas in which the use of relay leads to a reduction of total resources used, even if there are two hops involved. One can easily understand that e.g. two transmissions with $Q A M 64 \frac{5}{6}$ require a factor of $\frac{4}{15}$ less resources than one transmission with $Q P S K \frac{1}{3}$. That is the reason why relaying can improve the spectral efficiency of the cell.

\section{B. OFDMA Channel Access and Relaying}

One of the advantages of OFDM is that each subcarrier can have a different PhyMode (adaptive modulation and coding, AMC [2]), so a robust $Q P S K \frac{1}{3}$ can be used on frequencies where the channel is currently bad due to fading, while $Q A M 64 \frac{5}{6}$ can be used on more stable subcarriers with higher $S I N R$ values.

Beyond the classical OFDM, OFDMA means that within one OFDM symbol, several different UT receivers can be addressed. Dynamic subcarrier assignment (DSA) selects the best resources for each UT based on channel state information (CSI). As the distance and pathloss may be very different among the UTs, it is likely that the PhyModes used for each UT are very different within the full OFDM symbol. The granularity of these resources is typically a chunk, which is a group of subcarriers if $f$-direction and a number of OFDM symbols in $t$-direction. Figure 2(a) shows the frame format for 3GPP-LTE. A chunk of size 12 subcarriers times 6 effective symbols (gross 7) can carry up to 360 bits in PhyMode $Q A M 64 \frac{5}{6}$. Resources used at one location (cell) can be reused at another place (see frequency reuse distance $=$ cluster order 3 in figure 1 left). But also within one cell, there can be a spatial reuse by using beamforming, multipath transmission and spatial multiplexing by the use of distributed antenna elements.

\section{FDD Mode Multihop Operation}

The duplex mode decision, i.e. how to separate uplink from downlink traffic, is just a use of orthogonal resources, i.e. non-interfering blocks are assigned. In TDD, downlink and uplink phases alternate periodically, for example in the Winner system [8]. Thus there is the need for a guard time for TDD, which we don't need in FDD. In FDD we also never have 


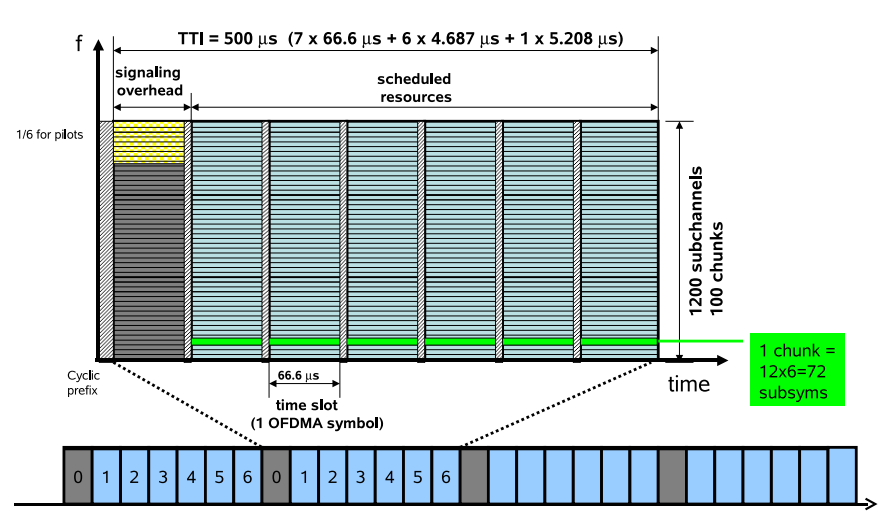

(a) One Downlink TTI frame

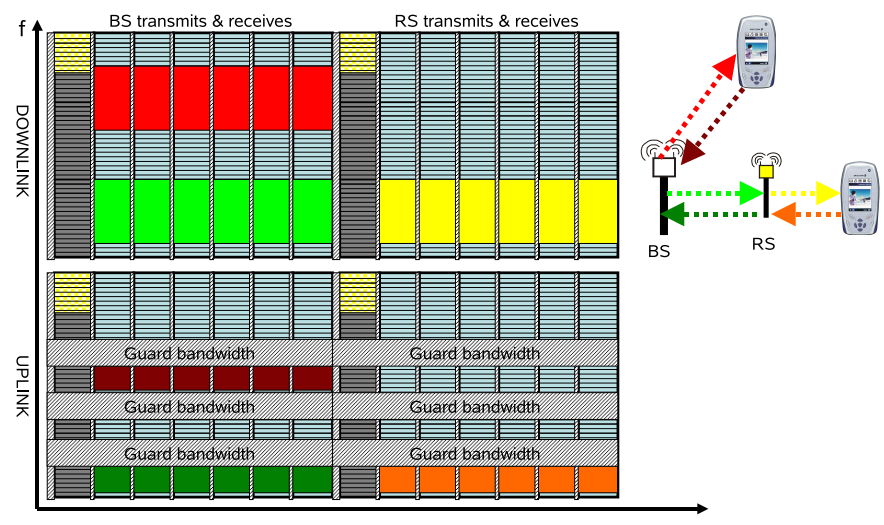

(b) DL and UL frames for relaying

Fig. 2. Left: TTI Frame format for 3GPP-LTE [1] $(500 \mu s)$. Right: Resource allocation for two hops.

interference among DL and UL transmissions. The difference between singlehop and multihop is that from time to time a complete frame is allocated to the second hop. The multihop PDU transport over the wireless loop has four phases for which it takes four frames: $B S \rightarrow R N, R N \rightarrow U T, U T \rightarrow R N$ and $R N \rightarrow B S$.

The smallest granularity resource unit (fig. 2(a)) is a chunk, and 100 of them fit into this basic frame. Symbols used for signaling and synchronization are out of focus here (shown in grey) and the only impact here is the overhead ( $\frac{1}{7}$ of the resources are not available for data throughput). Downlink and uplink transmissions happen simultaneously in the same frame raster. There are three ways to integrate relaying, i.e. the frames used for the second hop (or beyond):

- Time Domain Relaying: resources for hop 1 and hop 2 are separated in time (sequentially)

- Frequency Domain Relaying: resources for hop 1 and hop 2 are separated in frequency (neighbour band)

- OFDMA Domain Relaying: resources for hop 1 and hop2 are separated in frequency (subchannels)

Frequency Domain Relaying is trivial. It simply means we need another center frequency for the second hop. Since this wastes a lot of valuable spectrum, it is abandoned.

\section{Time, OFDMA and Space Domain Relaying}

For a normal singlehop transmission (upper terminal in figure 2(b)) the BS uses dedicated resources on the downlink channel which it can allocate for itself, knowing the traffic demand. Transmissions to and from the RN happen in the same frame, just like with any UT. For the second hop DL transmissions, the RF sender of the RN is active. The transmission must happen in a separate frame to avoid interference and the BS must reserve the complete resources of this frame for the second hop.

With OFDMA there are some additional benefits for relaying: The resources can be subdivided in a finer granularity than what would be possible with OFDM only. Figure 2(b) shows that first-hop transmissions are all treated the same way. They just occupy the required resources for their traffic. There is no waste due to completely assigned but incompletely used frames. In the uplink also several UTs share the full bandwidth, each of them transmitting on a subset of subchannels. The $\mathrm{BS}$ or RN coordinates the orthogonal non-interfering use of these subchannels by the UTs. A power control and exact synchronization is required so that neighboring resources on the UL can be used by independent senders.

Space division multiplex (SDM) techniques can also be utilized to have more orthogonal resources to offer. In multihop scenarios the BS and RNs must have separate resources in time and frequency, but the RNs among themselves and their UTs might be a suitable distance away to let them transmit on the same resource, only separated in space. The intracell interference must be accounted for, but the benefit is an improved spectral efficiency in the cell. This is especially effective in non-line-of-sight (NLOS) conditions like assumed here, with strong pathloss or many heavily shadowed areas. This parameter SDM vs. TDM is evaluated in the next section.

\section{Multihop Performance}

The basic scenarios for relaying (fig. 1) have been investigated. The results of an analytical/numerical analysis are shown now, For the link level function [9] between $S I N R$ and potential data rate for each PhyMode we use the recent MI (mutual information) approach with a closed formula [10]. The function in Figure 3 shows its performance. The numeric evaluation has been performed with own code in Matlab.

\section{A. MAC Layer Analytical Model}

These steps are calculated in order to get the MAC throughput results.

- Transmit Power: $37 \mathrm{dBm}$ at the BS, $34 \mathrm{dBm}$ at the RN,

- Bandwidth: $b=18 \mathrm{MHz}$ net (20MHz system),

- Pathloss I: non-line-of-sight propagation,

- Pathloss II: slow and fast fading effects [11],

- Interference: neighbor cell BSs interfere (100\% load, cluster order $C=7$ ),

- Noise: accounted for but not serious in interferencelimited systems,

- SINR: the first performance measure below PHY layer, 
- MI: mutual information determined from $S I N R$ and modulation ( [10]),

- BER: bit error ratio, the PHY performance result, depends on the Channel coder used,

- PER: packet error ratio, the result after channel decoding $\left(P E R=1-(1-B E R)^{N / b i t s}\right)$,

- Delay: determined by $P E R$ (ARQ retransmissions) and roundtrip times $\left(d_{\text {above } A R Q}=T_{\text {frame }} \cdot(1-P E R)^{-1}\right)$,

- Throughput: determined by bandwidth, PhyMode (modulation and code rate) and ARQ overhead (Eq. 4).

- Second Hop Throughput: reduced by resources required on first hop (Eq. 5).

- Capacity: integrated over the cell area (Eq. 3).

The system capacity is determined by assuming equal traffic load for each user terminal and a homogeneous user density over the area. This means that a UT far outside, having a low PhyMode, requires more share of the resources than a UT close to the BS. The following equation [12] for the capacity $c$ considers this:

$$
\frac{1}{c}=\int_{\text {cellarea }} \frac{1}{\text { Capacity }(x, y)} d x d y
$$

The capacity $c$ in bit/s can be used to calculate the spectral efficiency $e=c / b$ using the bandwidth $b$. On DLC (MAC) layer, there is an overhead due to framing, signaling and ARQ retransmissions. The latter depends on PER, which can be taken into account when assuming selective repeat ARQ by equation 4 .

$$
r_{\text {above } A R Q}=r_{\text {belowARQ }} \cdot(1-P E R)
$$

The different PhyModes determine the maximum rate $r_{i, \max }$ usable on each hop. We can get the maximum rate on the second hop to be

$$
r_{2}=\left(r_{1, \text { max }}^{-1}+r_{2, \text { max }}^{-1}\right)^{-1}
$$

For every location $(x, y)$ we can now determine the best rate out of $r_{1}, r_{2}$ and decide which is the "best server", the BS or one of the RNs. Depending on the parameters, the area served by RNs differs from case to case, which results in different numeric results for the system capacity and spectral efficiency. The analysis of these parameters is shown next.

\section{B. Results}

The parameters and their default values are

- cluster order: $C=7$

- spatial reuse: $T D M$ or $S D M$

- relay antenna gain: $G=14 d B$ or $G=0 d B$

- sectors per cell: $S=1$.

- coverage optimized (CO) vs. throughput optimized (TO) scenario

- directional antenna (DA, $A=\frac{4 \pi}{3}$ ) vs. omnidirectional antenna $(\mathrm{OA}, A=2 \pi)$

Figure 5(a) and 5(b) shows results for the coverage and capacity extension scenarios defined in fig. 1 . The circles notate the cell boundaries with given radius $R=1600 \mathrm{~m}$ here. The best station (best server) is determined by the highest rate which any of the stations can offer. The rate/throughput

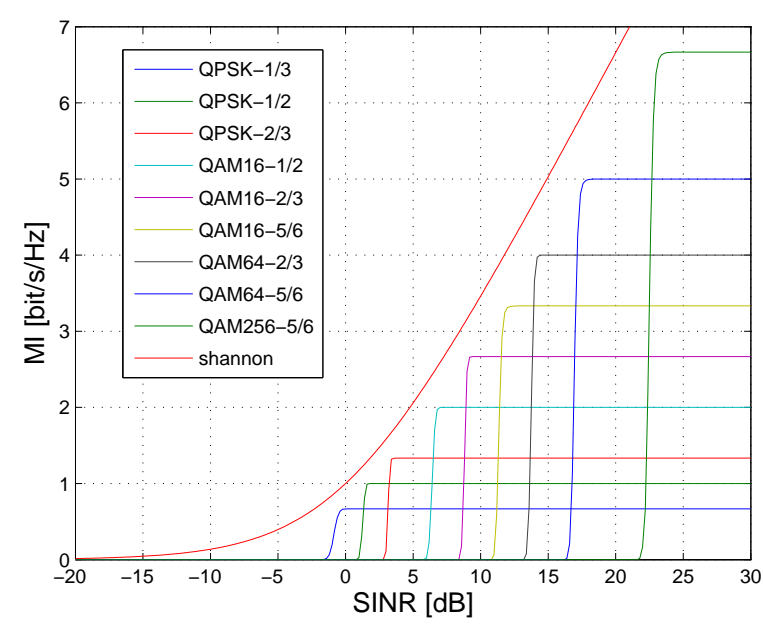

Fig. 3. Link level performance for different PhyModes

results contain the maximum achievable rate at a certain position within the cell, taking also the required first hop resources into account. The relay is chosen as the serving station (association) if this is an advantage for the cell (less resources used), which is here the same as the maximum rate. In both scenarios there are huge areas where the relay offers an advantage over the singlehop case. For the default parameters in Figure 5(a) we observe that the inner hexagon is completely served by the BS. The 240 degree directional antenna of RNs is adjusted to radiate only into the outer hexagons. This is the area which is fully served by $\mathrm{RN}$ as the best server. So the coverage extension of $+200 \%$ compared to a singlehop system is proven. In Figure 5(b) we see that an outer ring of approx. $30 \%$ within the hexagon is best server by RNs. This region near the cell border is no longer an area of the worst PhyMode, the highest (transmit) power consumption and the worst performance, due to the RNs. We obtain the performance results in Table II for these two scenarios under "Throughput TDM DA G14" and "Coverage TDM DA G14". Figure 4 additionally shows the SINR and resulting MAC data rate for the coverage scenario. As we observe, the SINR might be as high close to the RNs as to the BS, but the maximum rate around RNs is reduced due to the resources required for the first hop.

If we now switch on SDM, i.e. let the RNs transmit and receive to their UTs in parallel and account for their interference, the results in Figure 5(c) comes out for the coverage scenario. Figure 5(d) shows SDM for the throughput enhancement scenario.

The results above were using directional $A=\frac{4 \pi}{3}$ antennas in the RNs which point to the outside of the cell. This is especially useful to reduce SDM intra-cell interference. To see the contrast, Figure 5(e) and Figure 5(f) show the TDM and SDM results with omnidirectional antennas respectively. Compared to the directional antennas we observe a bigger area covered by the RNs at the edge of the inner hexagon and for SDM a bigger interference-problematic area between the RNs. 


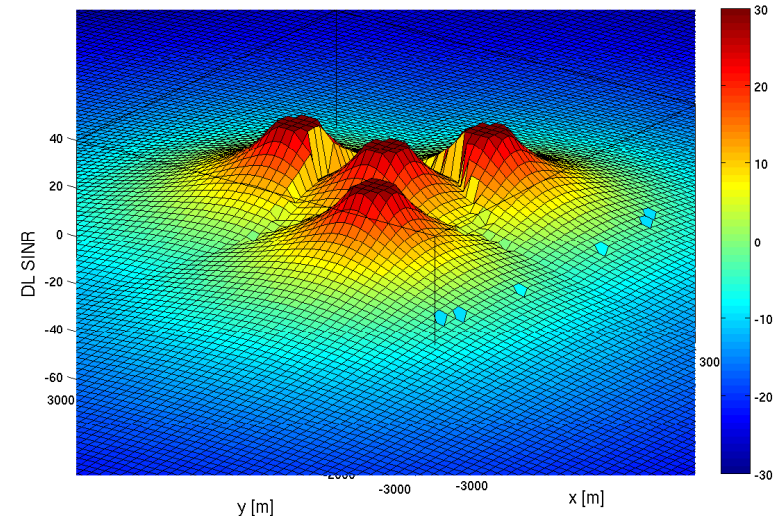

(a) Coverage Extension Scenario: SINR(area)

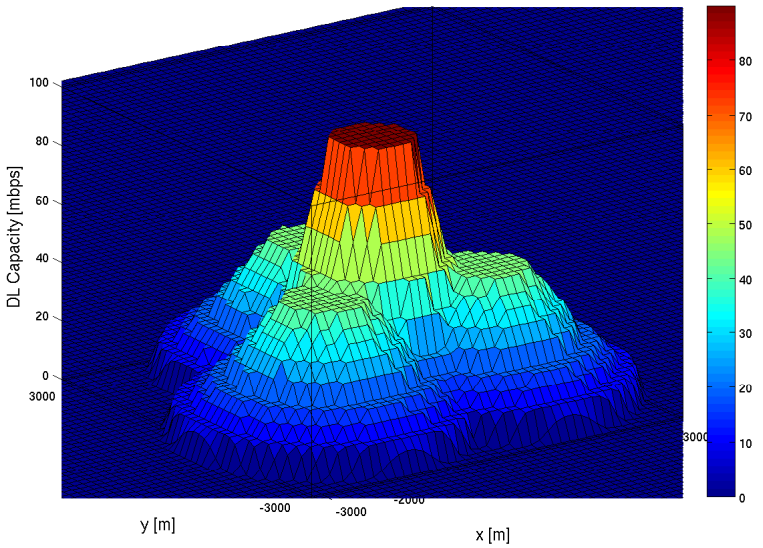

(b) Coverage Extension Scenario: Rate(area)

Fig. 4. Results for the two basic relaying scenarios. In the graphs, a DL capacity close to $100 \mathrm{Mbit} / \mathrm{s}$ in the center is achieved in both scenarios.

Results without antenna gain between $\mathrm{BS}$ and $\mathrm{RN}$ are not shown graphically, but numerically in table II. The main points about the parameters are: An antenna gain (G14) for the stationary first hop link should be possible. Directional RN antennas trade off some less area near the center against more area between RNs, but the spectral efficiency doesn't change much. Using space division (SDM) resource reuse among RNs we gain a lot of capacity, especially in the coverage scenarios.

\begin{tabular}{|l|l|l|}
\hline Scenario & $\begin{array}{l}\text { DL Capacity } \\
{[\mathrm{bit} / \mathrm{s}]}\end{array}$ & $\begin{array}{l}\text { DL SpecEff } \\
{[\mathrm{bit} / \mathrm{s} / \mathrm{Hz}]}\end{array}$ \\
\hline Throughput TDM DA G14 & 50973029 & 2.8318349554 \\
\hline Throughput SDM DA G14 & 58642110 & 3.2578950216 \\
\hline Throughput TDM DA G0 & 48737548 & 2.7076415712 \\
\hline Throughput TDM OA G14 & 50730651 & 2.8183695086 \\
\hline Throughput SDM OA G14 & 53799503 & 2.9888612873 \\
\hline Coverage TDM DA G14 & 34087801 & 1.8937667754 \\
\hline Coverage SDM DA G14 & 64801466 & 3.6000814652 \\
\hline Coverage TDM DA G0 & 21385809 & 1.1881005356 \\
\hline Coverage TDM OA G14 & 34937225 & 1.9409569593 \\
\hline Coverage SDM OA G14 & 65309985 & 3.6283325128 \\
\hline
\end{tabular}

TABLE II

DOWNLINK CAPACITY AND SPECTRAL EFFICIENCY OF THE DISCUSSED SCENARIOS

\section{CONCLUSION}

This paper treats the properties and MAC performance of a multihop FDD mode cellular system. Two basic scenarios, the Coverage Extension Scenario and the Capacity Extension Scenario, are analyzed for relaying under various parameters. Multihop communication can provide an remarkable increase in link and network capacity, especially if we utilized possible antenna gains, directional antennas, and possible parallel transmissions of remote relays (SDM). The most important fact is that no fibre line access is required for relays and relay devices can be cheaply installed anywhere. If the association decision is done the right way by minimizing used resources, there will be always an advantage of having relays instead of base stations only. As result, the total spectral efficiency rises and not only area coverage but also the average system capacity is increased over the area.

\section{REFERENCES}

[1] http://www.3gpp.org/Highlights/LTE/LTE.htm.

[2] G. Song and Y. Li, "Utility-based resource allocation and scheduling in OFDM-based wireless broadband networks," IEEE Communications Magazine, pp. 127-134, Dec 2005.

[3] R. Pabst, B. Walke, D. C. Schultz, and et al, "Relay-Based Deployment Concepts for Wireless and Mobile Broadband Radio," IEEE Communications Magazine, pp. 80-89, Sep 2004.

[4] B. Walke and R. Briechle, "A local cellular radio network for digital voice and data transmission at 60ghz," in Proceedings Cellular \& Mobile Comms. Intern. London: Online, Nov 1985, pp. 215-225. [Online]. Available: http://www.comnets.rwth-aachen.de

[5] R. Schoenen, R. Halfmann and B. Walke, "An FDD Multihop Cellular Network for 3GPP-LTE," in Proceedings of the VTC Spring Conference, Singapore, May 2008. [Online]. Available: http://www.comnets.rwth-aachen.de

[6] A. Otyakmaz, R. Schoenen, and B. Walke, "Parallel Operation of Halfand Full-Duplex FDD in Future Multi-Hop Mobile Radio Networks," in Submitted to the European Wireless Conference, Prague, Jun 2008.

[7] N. Esseling, H. Vandra, and B. Walke, "A forwarding concept for hiperlan/2," in Proceedings of European Wireless 2000, Sep 2000, pp. 13-18. [Online]. Available: http://www.comnets.rwth-aachen.de

[8] W. Mohr, "The WINNER (Wireless World Initiative New Radio) Project - Development of a Radio Interface for Systems beyond 3G." in Proc. of IEEE Personal Indoor and Mobile Radio Conference 2005 (PIMRC05), Berlin, Germany, Sep 2005.

[9] M. Ahmed, H. Yanikomeroglu, and S. Mahmoud, "Fairness enhancement of link adaptation techniques in wireless access networks," in Proc. Vehic. Technol. Conf.-Fall, 6-9 Oct. 2003, pp. 1554-1557.

[10] R. Schoenen and B. Walke, "On PHY and MAC performance of 3G-LTE in a multi-hop cellular environment," in Proceedings of the 3rd IEEE International Conference on Wireless Communications, Networking and Mobile Computing (WiCOM), Shanghai, China, Sep 2007. [Online]. Available: http://www.comnets.rwth-aachen.de

[11] M. Einhaus, O. Klein, B. Walke, and R. Halfmann, "Simulative mac level performance evaluation of an ofdma system under the consideration of frequency correlated fading," in Proceedings of the 2007 IEEE Wireless Communications and Networking Conference, Hong Kong, Mar 2007, p. 6. [Online]. Available: http://www.comnets.rwth-aachen.de

[12] C. Hoymann and S. Goebbels, "Dimensioning cellular wimax part i: Singlehop networks," in Proceedings of European Wireless 2007, Paris, France, Apr 2007, p. 7. [Online]. Available: http: //www.comnets.rwth-aachen.de 


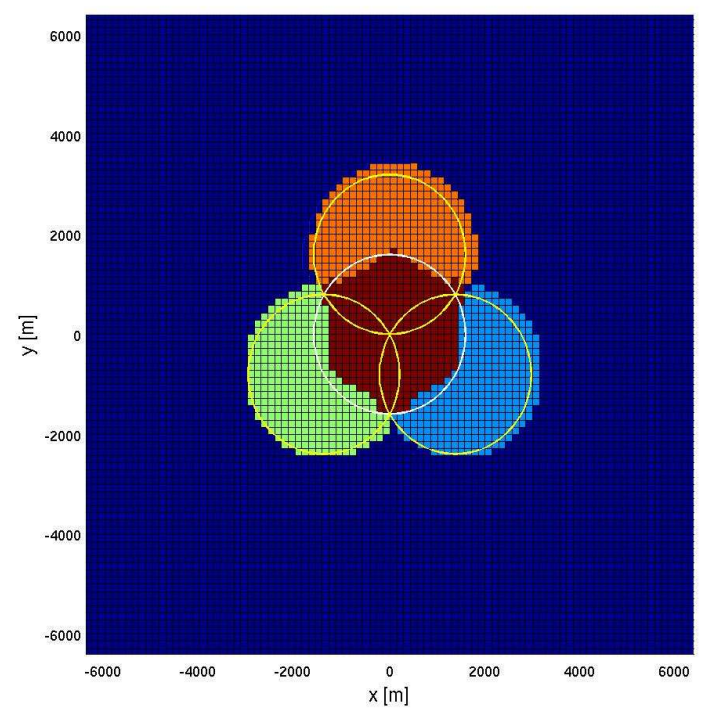

(a) Coverage Extension Scenario: Best Server(area)

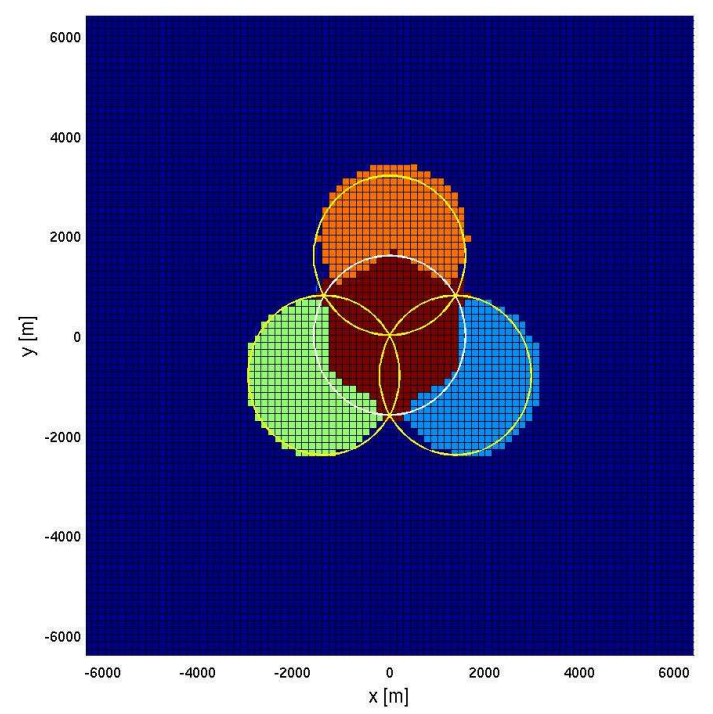

(c) Coverage Extension Scenario using SDM, $A=\frac{4 \pi}{3}, G=14 d B$

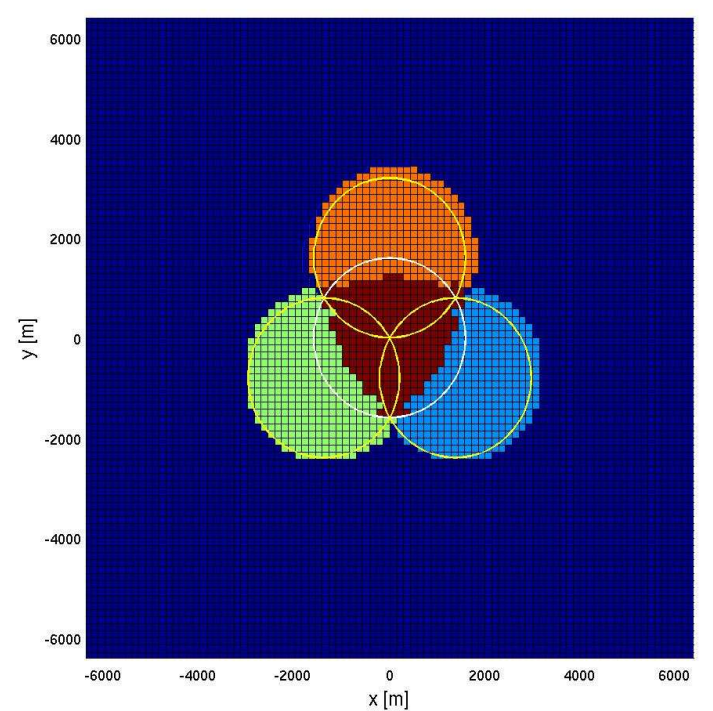

(e) Coverage Extension Scenario using TDM, $A=2 \pi, G=14 d B$

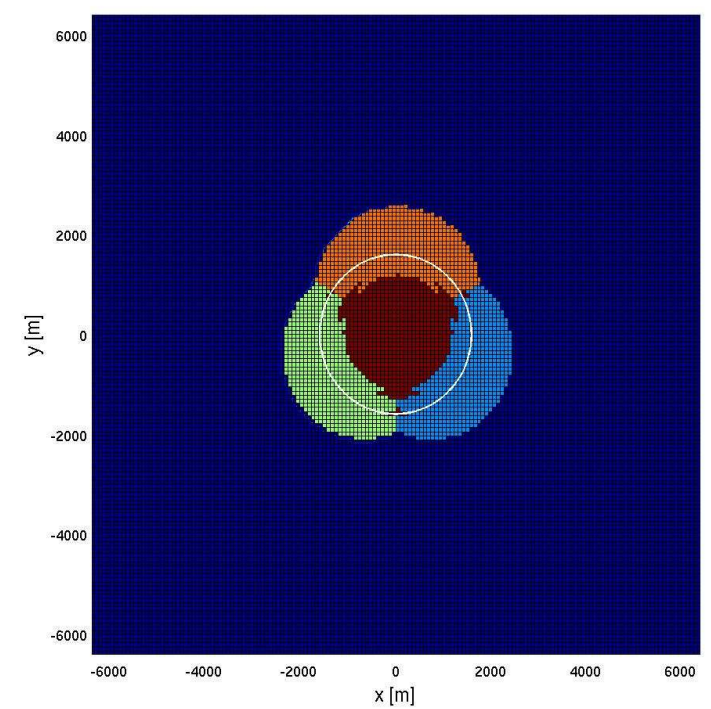

(b) Capacity Extension Scenario: Best Server(area)

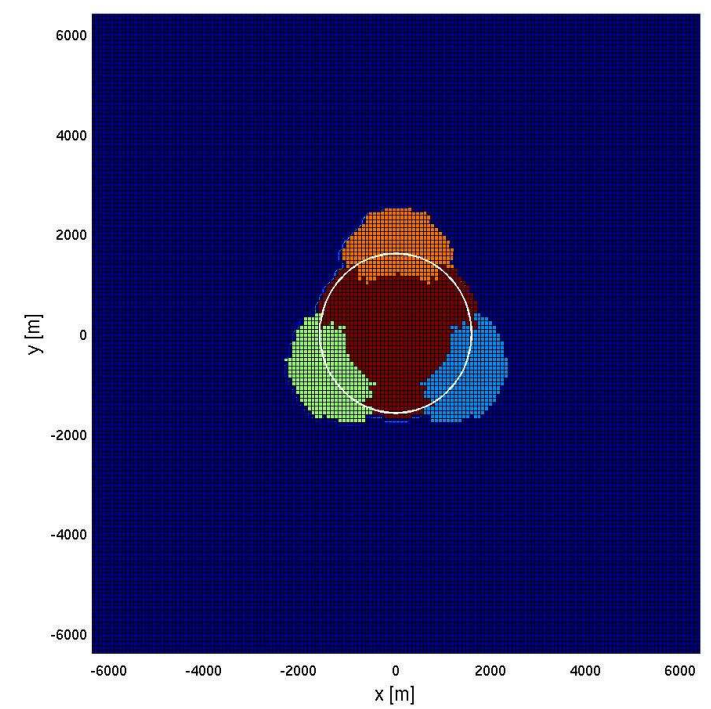

(d) Capacity Extension Scenario using SDM, $A=\frac{4 \pi}{3}, G=14 d B$

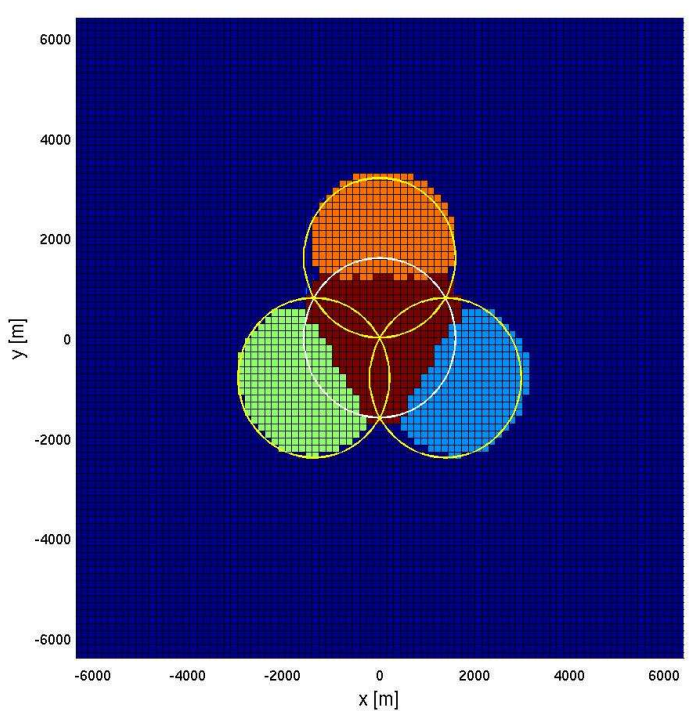

(f) Coverage Extension Scenario using SDM, $A=2 \pi, G=14 d B$

Fig. 5. Best station results over the cell area evaluated for several parameters 